\title{
The perception of an opening from expanding motion
}

\author{
HIROYUKI ITO \\ Kyushu Institute of Design, Fukuoka, Japan
}

\begin{abstract}
The ability of humans to detect an opening's 3-D structure from expanding motion was tested. Computer simulations of dotted tunnels were used to generate optical flows typically encountered when one moves through an opening. Experiment 1 qualitatively tested the ability to detect the shape of a tunnel's vertical section. The observers could choose the correct shape for each of seven simulated shapes. The percentages of correct responses were much higher than those under static conditions. Experiment 2 tested whether or not one could quantitatively detect the vertical-horizontal proportion of the elliptic tunnels. The result shows quite high correlations $(r=.93 \sim .97)$ between perceived proportions and simulated ones. The slopes of the regression lines were around 1.0. Experiment 3 investigated the necessary stimulus duration for detecting an opening's shape. Relative size (width and height) was significantly detected under four-frame (72.7-msec) conditions by 3 out of 4 subjects. The other subject performed well under eight-frame conditions. These results indicate that the human visual system can instantly detect the 3-D structure of an opening surrounded by objects from expanding optical flows while one is in forward motion.
\end{abstract}

It is important for humans or animals to analyze expanding optical flow because it offers information concerning approaching objects. In given situations, they have to change their posture or the direction of locomotion to avoid collision with obstacles. Researchers seem to have emphasized the role of expanding motion, such as in the detection of an approaching object. In fact, Schiff, Caviness, and Gibson (1962) demonstrated that "looming" is a specific and meaningful pattern that suggests collision. A "looming detector" (Regan \& Beverley, 1978a, 1978b) is considered to be useful for the task. In addition, as an example of higher order functions, Todd (1981) and Simpson (1988) demonstrated that observers could detect which of two objects would collide with them from expanding images.

However, expanding motion does not always mean collision with an obstacle. For example, expanding motion that arises while an observer is moving forward through an opening has the opposite meaning. Despite global expanding motion, keeping the self-position in the center of the opening enables one to move forward safely, without collision (e.g., by "steering"; see Gibson, 1979). Stepping aside may even cause a collision with an obstacle to one's side. Thus, for safe locomotion, one may also have to detect the 3-D structure of the surround from expanding motion in addition to expanding motion itself.

This research was supported by scientific research funds from the Ministry of Education in 1991. I would like to thank Christopher J. Savoie for his assistance in manuscript preparation. Correspondence concerning this article should be addressed to H. Ito, Department of Visual Communication Design, Kyushu Institute of Design, 4-9-1, Shiobaru, Minami-ku, Fukuoka, 815 Japan (e-mail: ito@kyushu-id. ac.jp).
This aspect is associated with "structure-from-motion" problems-that is, with how we detect the 3-D structure of an environment from changing 2-D images. Some have approached this problem by utilizing images under parallel projection (e.g., Ullman, 1979, 1984; Wallach \& O'Connell, 1953). However, such an approach raises some problems for dealing with motion in depth, because expanding or contracting 2-D image motion representing motion in depth arises only under polar projection. Gibson (1950) adopted polar projection and introduced certain kinds of optical flow patterns, including expansion while an observer is moving forward. One of those patterns, motion parallax in a horizontal orientation, has been experimentally proved to be available in the perception of 3-D structure of a surface (Rogers \& Graham, 1979; see also Braunstein \& Tittle, 1988; Ono \& Steinbach, 1990). On the other hand, the perception of 3-D structure from an observer's or object's translation along the line of sight (which produces expanding motion) has not been given much attention.

Andersen (1989) reported that human observers could detect up to three dot planes moving in depth even when all the dots were displayed on the same display screen. Since adjacent dots could belong to different depth planes and there was no smooth overall velocity distribution, which plane a dot belonged to depended on the global expansion rates of the planes. Ito and Matsunaga (1990) also showed that human observers could detect the relative distance of two simulated walls from expanding motion generated by walking forward. They suggested that differences in expansion rates indicate depth and demonstrate the possibility of perceiving 3-D structure of surfaces from expanding motion. However, these reports referred only to either 
depth stratification of frontal parallel planes or the depth order.

The present study focuses on the ability to detect the "shape" of an opening from expanding motion. The "shapes" here are different from the "depth" referred to in the reports above. The "depth" is along the line of sight, whereas "shapes" here are at right angles to the line of sight. This ability was tested, qualitatively and quantitatively, in three experiments by using random dot simulations of tunnels. The necessary stimulus duration was also measured. This is one of the important characteristics of this task, because the observer must instantaneously decide the appropriate direction of locomotion in order to avoid collision. The relationship between the observer and the environment changes according to locomotion, over time.

\section{EXPERIMENT 1}

\section{Method}

Subjects. Four subjects participated in Experiment 1. One was the author; the others were graduate students at the Kyushu Institute of Design.

Apparatus and Stimuli. Computer simulations of dotted tunnels through which an observer might move were used as stimuli (see Figure 1). Structurally, a tunnel is a type of opening surrounded by obstacles. The use of tunnel simulations had an advantage: Although the stimulus was a motion display, the 3-D structure (including shape, position, and orientation) could be kept constant during presentation. The actual stimuli were patterns of random dots that moved radially.

The displays were generated by a microcomputer (Sharp CZ644C) and presented on a monitor display (Sharp CZ-614D). Motion displays were created by sequential presentation of bright dot patterns $\left(8.4 \mathrm{~cd} / \mathrm{m}^{2}\right)$ and were refreshed at $55 \mathrm{~Hz}$. The size of the display screen was about $18 \mathrm{~cm}$ vertically and $26.2 \mathrm{~cm}$ horizontally. Stimulus dots were presented in an annulus display window, shown in Figure 2, which had a black background. The display window was located between the inner circle, which had a diameter of $8.8^{\circ}$ of visual angle, and the outer one, which had a diameter of $26.5^{\circ}$ of visual angle. The nondisplay area of the screen was colored dark blue $\left(.02 \mathrm{~cd} / \mathrm{m}^{2}\right)$.

The center opaque disk occluded the cluster of bright dots caused by perspective transformation. This occlusion was also useful for masking zigzag motion in the center field, which would arise from the interaction between the low resolution ( 768 dots

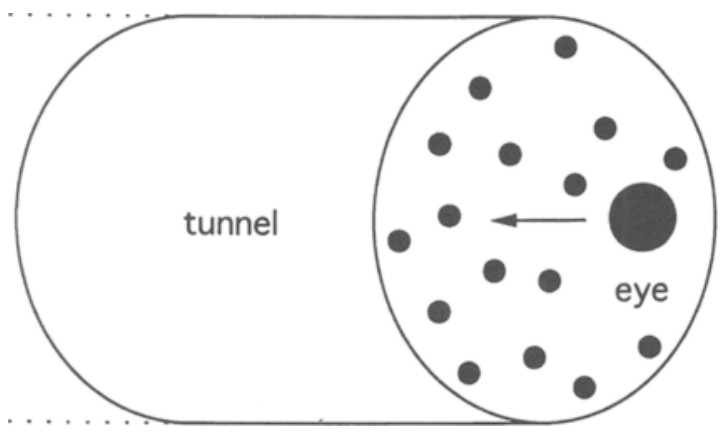

Figure 1. Simulated situation in Experiment 1. This represents a situation in which an eye moves forward through a dotted tunnel.

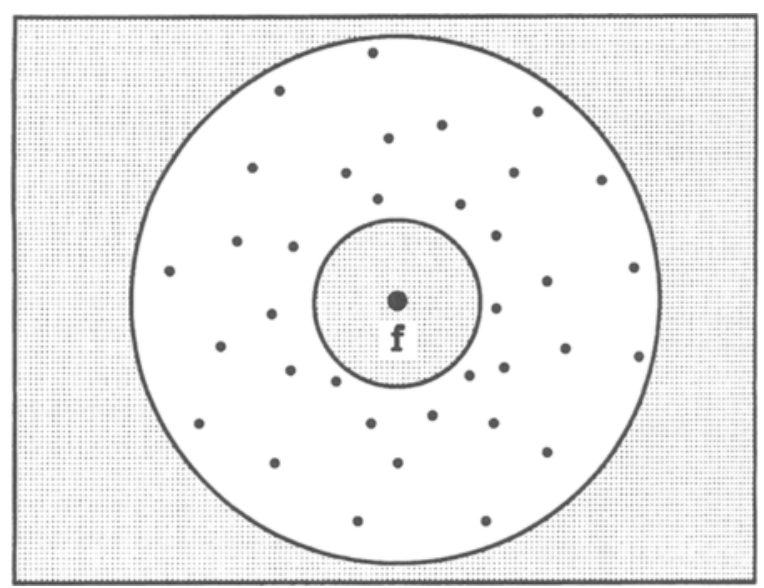

Figure 2. Display window. $f$ in the center indicates the fixation point. The actual display window was the region between the inner and outer circles.

horizontally $\times 512$ dots vertically) of the graphic system and the fine position changes of the dots because of the large simulated distance.

The shapes of the simulated tunnels' vertical sections were varied (square, rotated square, circle, triangle, rotated triangle, vertical ellipse, or horizontal ellipse) as in Figure 3. The simulated diameter of the tunnel under Condition $C$ was $200 \mathrm{~cm}$ and the approximated speed of moving through the tunnels was $257.8 \mathrm{~cm} / \mathrm{sec}$. The area of the vertical section was the same under all conditions. The number of dots was 400 in all the conditions, but the number of visible dots was somewhat less because unseen dots were positioned outside the window. The farthest dot distance simulated was $15 \mathrm{~m}$, but because of the center occlusion, the farthest parts of the tunnels were not seen.

Dots were distributed on the surfaces of the simulated tunnels. Within the display window, there was a slight gradient of dot density from the inner to the outer parts, caused by perspective transformation. This density gradient was not critical, however, because all the stimulus displays had the same gradient component that indicated depth along the line of sight. However, another type of density gradient that could indicate the tunnels' shapes was possible. When dots were distributed in equal density on the surfaces of the simulated tunnels, dots projected on the display screen would gather in some locations, as, for example, at each corner of the triangle. In order to reduce this effect on perceived shapes, the distribution of dots was controlled, as follows: First, the annular display screen was divided into 400 equal fan shapes; then, one dot was distributed to each fan area. This manipulation was useful for decreasing density differences between orientations that could serve as cues to the tunnel's shape.

In order to make sure that the expanding motion itself, not texture gradient, was effective for perception of the tunnels' shapes, static conditions were also included. The patterns in the static conditions were randomly selected from the sequential patterns of the corresponding motion displays.

Procedure. Before the experiment began, the subjects were informed that the patterns were simulations of movement through tunnels. Each trial began with the presentation of a fixation point centered on the screen. After a few seconds, a motion display appeared in the display window for $2 \mathrm{sec}$ and then disappeared. Subjects chose whichever one of seven shapes (shown in Figure 3) drawn on a sheet that appeared to be most consistent with their perceived structure of the tunnel at the point of vertical section. No feedback was given. Subjects watched the display with one eye 

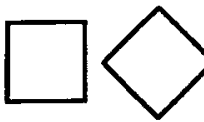

$\mathbf{s}$

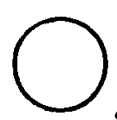

C

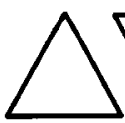

$\mathbf{T}$

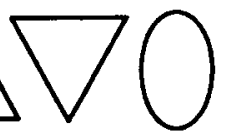

Tr
E V

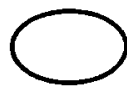

E h

Figure 3. Seven simulated shapes of the tunnel's vertical section. The shapes were varied, but the area was the same. $S$, a square; $\mathrm{Sr}$, a square rotated $45^{\circ} ; C$, a circle; $T$, a triangle; $T r$, a triangle rotated $180^{\circ} ; \mathrm{Ev}$, a vertically long ellipse; and $\mathrm{Eh}$, a horizontally long one.

from a distance of $37 \mathrm{~cm}$, through a box that eliminated the light from outside and restricted head movement.

The experimental session was divided into four blocks, each of which included 70 trials ( 7 simulated shapes $\times 2$ motion conditions $\times 5$ repetitions). Five-minute intervals occurred between blocks. The order was randomized within each block. Before the experimental session, there was one block of training trials.

\section{Results and Discussion}

Figure 4 shows the percentages of correct responses. "Correct" means that the subject's perception corresponded with the simulated shape. In general, the percentages of correct responses under motion conditions were much higher than those under static conditions for all the subjects.

Under static conditions, Subject H.N. could not detect the S, Sr, and C shapes. For Subject N.K., only "circle" responses showed a very high "correct" percentage; he could not discriminate any shapes in the static displays,

so he chose "circle" in almost all the trials. This analysis was confirmed by his verbal report after the session. The other 2 subjects (H.I., K.S.) were able to detect the shapes with higher percentages than had been expected, even under static conditions. However, under motion conditions, their responses were almost perfect. It seems clear that expanding motion can effectively contribute to the detection of tunnels' shapes.

Alternative explanations may be possible. The number and positions of discontinuity in velocity distribution on the screen could be a clue to shape discrimination. However, three shapes had no discontinuity (circle, vertical ellipse, and horizontal ellipse), and the subjects could discriminate all three. Evidence against a necessity for discontinuity was also acquired in Experiment 2, in which only elliptic tunnels were used. Texture density information might still have been latent there and might have been activated during motion. Texture information did exist, because sensitive subjects could discriminate some of the shapes at some level even without motion, as noted above. Thus, in Experiment 2, the quantitative aspects of 3-D structure perception were investigated with texture density controlled more strictly.

\section{EXPERIMENT 2}

The purpose of Experiment 2 was to investigate how accurately human observers could detect the extent of an opening in terms of the proportion of height and width.

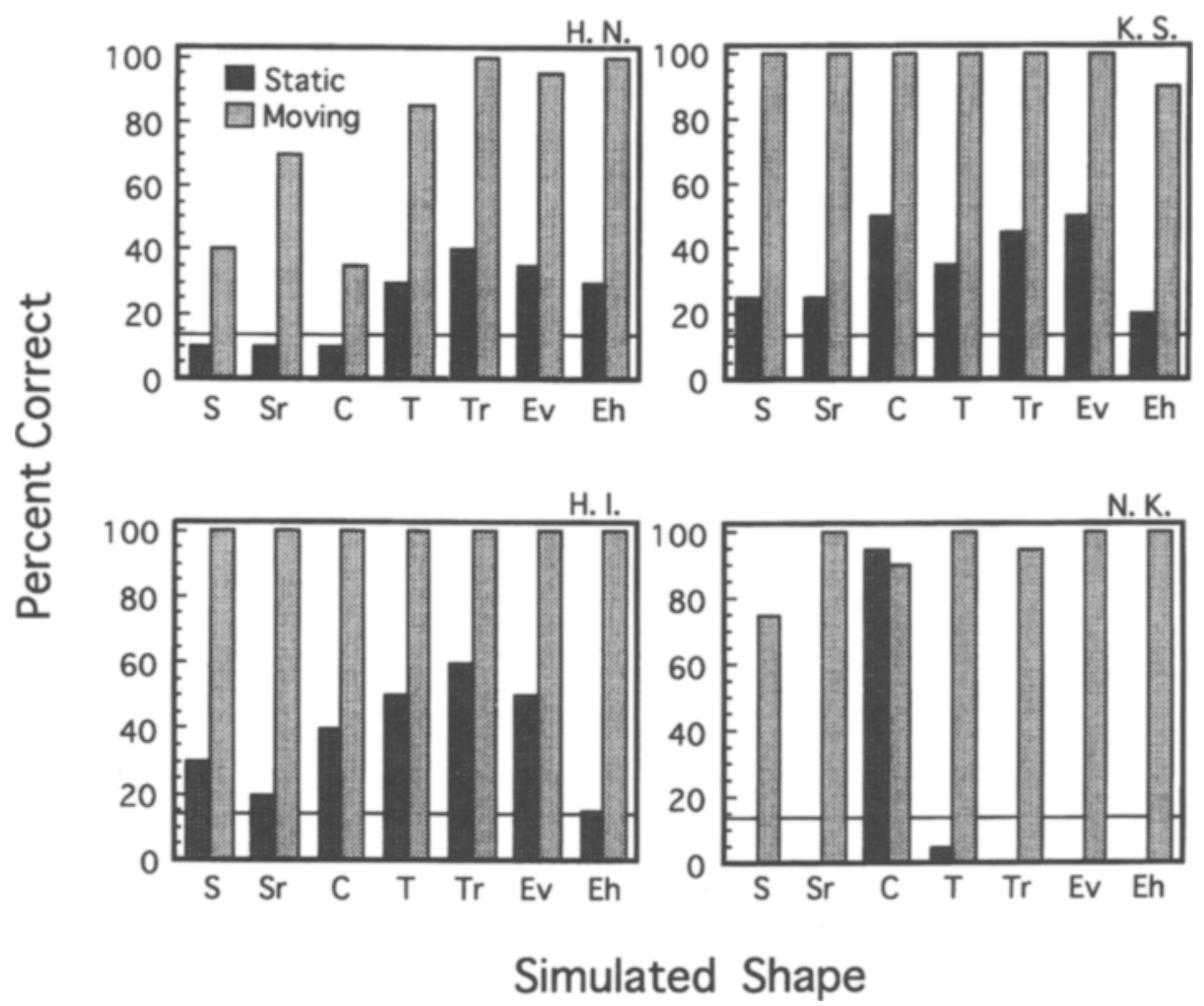

Figure 4. Percentages of correct responses. The results are shown individually. The horizontal line in the figure indicates the expected value. 

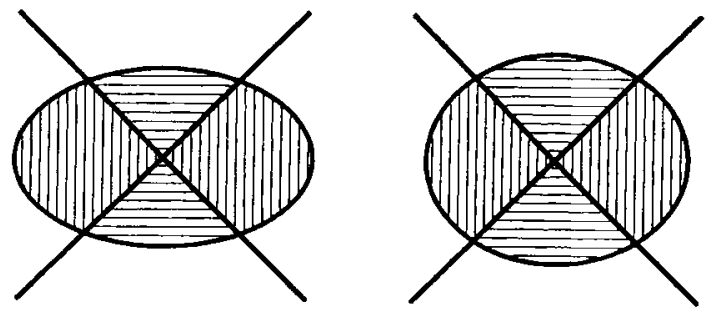

Figure 5. Controlling dot distribution. Each figure is one of the simulated shapes (vertical/horizontal $=0.6$ and 0.8 , respectively). In the left figure, 150 dots were assigned to vertical line areas and 250 dots to horizontal line areas. However, some dots were unseen, outside the annular display window, so the number of dots actually seen in the display window was almost equal for these areas. As for the right figure, with the same procedure, 184 dots were assigned to vertical line areas and 216 dots to horizontal line areas. Under vertically long conditions, the reverse distribution was assigned. Under the circular condition, there was no manipulation.

\section{Method}

Subjects. Three subjects took part in Experiment 2. One was the author; the others were graduate students.

Apparatus. The apparatus was the same as that for Experiment 1.

Stimuli. Computer simulations of the flow patterns that arise in one's moving forward through varieties of elliptic tunnels were used. All the ellipses had the same area, but the proportion of height and width was varied (vertical/horizontal $=1.67,1.25,1.0$, 0.8 , or 0.6 ).

The distribution of dots was controlled, as follows: first, the tunnels' elliptic shapes were divided into four fans as shown in Figure 5; then, appropriate numbers of dots were assigned to each region. This manipulation was done to cancel the inclination of dot distribution (the wider parts of tunnels would be dense on the 2-D screen).

The other conditions, as well as the display window, were the same as in Experiment 1.

Procedure. Subjects were told to choose 1 of 21 ellipses printed on a sheet, according to their perceived shape of the opening's vertical section. As can be seen in Figure 6, the shapes varied from horizontally wide (negative values) to vertically wide (positive values). The vertical/horizontal proportions in choices were decided to show a function of $1.07^{n}(n$ corresponds to each number in Figure 6).

There were four blocks in the experimental sessions, each of which included 40 trials: 5 simulated shapes $\times 2$ motion conditions (static and moving) $\times 4$ repetitions. The order was randomized within each block. Before experimental sessions, subjects tried one block as training. The other procedures were the same as in Experiment 1.

\section{Results and Discussion}

The results are shown in Figure 7. Under motion display conditions, the perceived proportions of height and width of the openings were almost perfectly consistent with the simulations for each subject. The correlations of coefficients were .95 (Subject K.S.), .97 (H.I.), and .93 (N.K.), and the regression coefficients were 1.02 (K.S.), 1.00 (H.I.), and .94 (N.K.).

On the other hand, under static conditions, all the subjects failed to detect the opening's shape. Results from 2 of the subjects showed no correlation between simulated and perceived proportion: - .05 (K.S.) and .00 (N.K.). Standard deviations under each condition (vertical bars in Figure 7) differed for these 2 subjects. K.S. may have tried to obtain the information of the shape from randomized distribution of dots but failed, and N.K. actually gave up attempting to detect the shapes. The results from Subject H.I. showed a slight correlation $(-.25)$, but it is impossible to explain the effect under motion display conditions from the correlation because the value was negative (i.e., in the opposite direction). This indicates that the dot distribution was over-controlled, and, consequently, this result may inversely emphasize the effect under motion display conditions.

It seems clear that the visual system can detect the shape of an opening quantitatively from expanding motion without texture information.

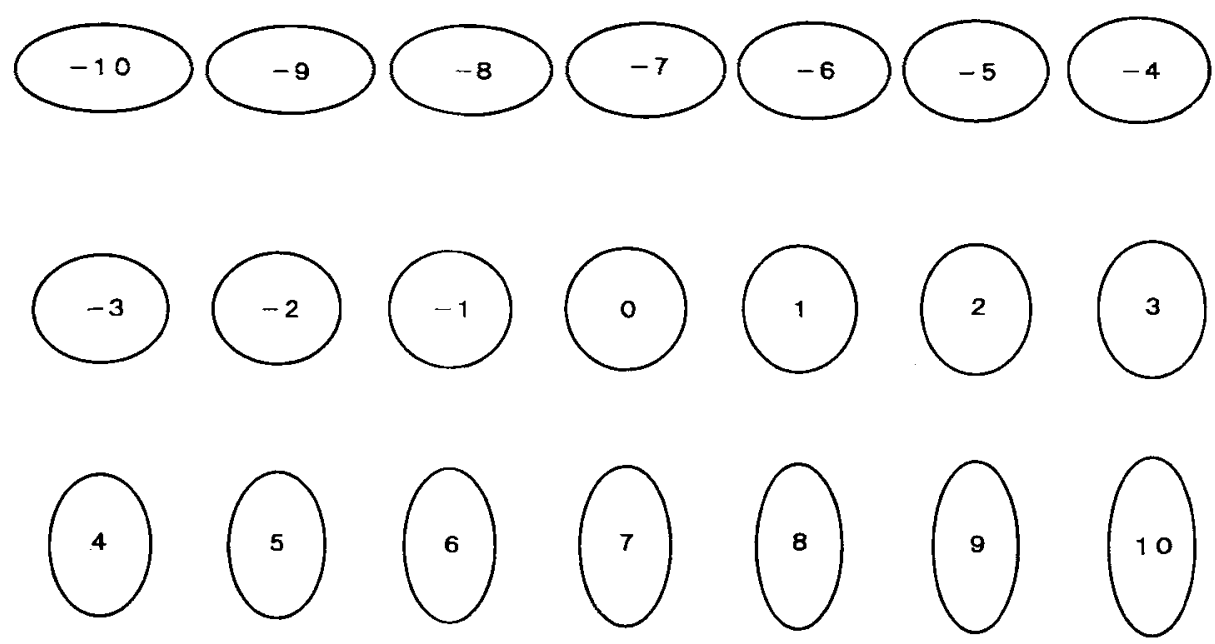

Figure 6. Subjects' choices. The value in each figure indicates the vertical/horizontal proportion $\left(1.07^{n}\right)$. The area was the same in all the ellipses. 


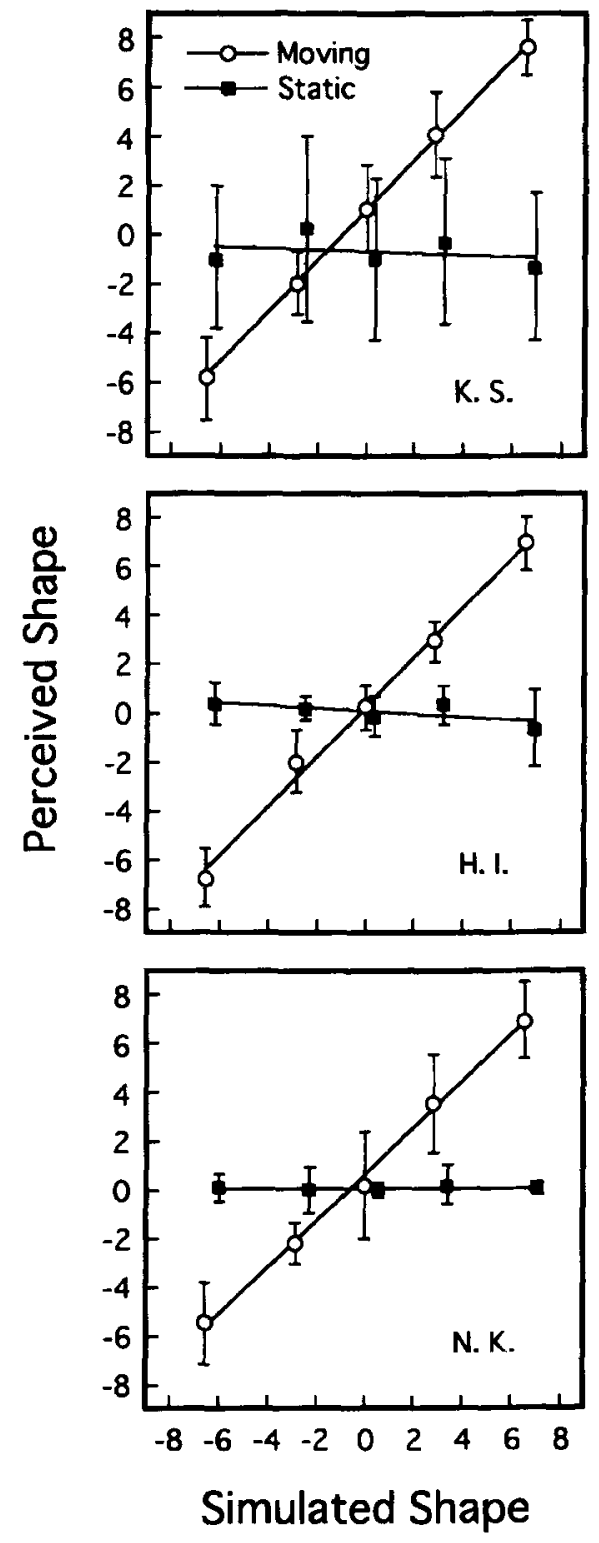

Figure 7. Perceived vertical/horizontal proportions, as a function of simulated ones. The solid lines in the figure represent regression lines under moving and static conditions. The slopes are almost 1.0 under moving conditions.

\section{EXPERIMENT 3}

The results of Experiments 1 and 2 demonstrate that the visual system can utilize the expanding motion in detecting an opening's shape. However, the detection cannot be useful for safe locomotion if it requires a large extent of time, because the relation between the observer and his/her surround continuously and rapidly changes according to his/her motion. The time required for the task was explored in Experiment 3.

\section{Method}

Subjects. Four subjects participated in Experiment 3. One was the author; the others were graduate students.

Apparatus and Stimuli. Two elliptical shapes of opening were simulated: horizontally wide (vertical/horizontal $=0.6$ ) and vertically wide (vertical/horizontal $=1.67$ ) proportions, chosen from those used in Experiment 2. The duration of presentation was varied: there were $2,4,8$, or 16 frames ( 1 frame corresponded to $18.2 \mathrm{msec})$. The simulated speed of moving forward was varied: there were high-speed $(257.8 \mathrm{~cm} / \mathrm{sec})$, low-speed $(128.9 \mathrm{~cm} / \mathrm{sec})$, or static $(0 \mathrm{~cm} / \mathrm{sec})$ conditions. The other conditions were the same as in Experiment 1.

Procedure. The task was to choose one of the two shapes (horizontally wide or vertically wide) according to one's perception and to report it verbally. If subjects couldn't detect the shape, the percentage of correct responses would be around 50 .

There were six blocks, each of which had 96 trials (4 duration conditions $\times 3$ speed conditions $\times 8$ repetitions). Each shape was assigned with the same likelihood. The order was randomized within each block.

\section{Results and Discussion}

The percentages of correct responses are shown individually in Figure 8. For all the subjects, eight frames of motion display seemed enough to detect the relative width between horizontal and vertical orientations. Even with four frames, correct responses from 3 subjects (K.M., S.F., and H.I.) were significant $(p<.01)$ under high-speed conditions, and, furthermore, 2 subjects' responses were significantly correct $(p<.01$ for S.F. and $p<.05$ for H.I.) under low-speed conditions. The fourframe durations lasted $73 \mathrm{msec}$. The changes of flow within this time duration correspond to those during one's moving through $9.4 \mathrm{~cm}$ (under low-speed conditions) or $18.8 \mathrm{~cm}$ (high-speed conditions) in depth. It seems possible to move the head through these extents of space even if the observer remains seated.

On the other hand, under static conditions, the percentages of correct responses were not significant $(p>$ .05 ). As a solitary exception, under the 2 -frame condition, correct responses from Subject S.F. were significant $(p<.05)$. This effect is difficult to explain, because his performance dropped in chance level under longer duration conditions. For the other subjects, under 2-frame conditions, the percentages of correct responses were around 50 as expected. For Subject N.K., under 4-, 8-, and 16-frame conditions, the percentages of correct responses were lower than 50. It seems that the effect of over-controlling dot distribution (as noted in Expriment 2) became clear under longer duration conditions.

It was expected that the performance would improve under high-speed conditions because the amount of flow changes over a given extent of time depend on the speed (consequently, distance). However, the effect of speed was not so clear, since it was clearly seen only under fourframe conditions and only for Subject H.I. (the author). If the effect can be found only around the threshold duration, the condition settings should be more strict (e.g., one should use a display system with a higher refresh rate 


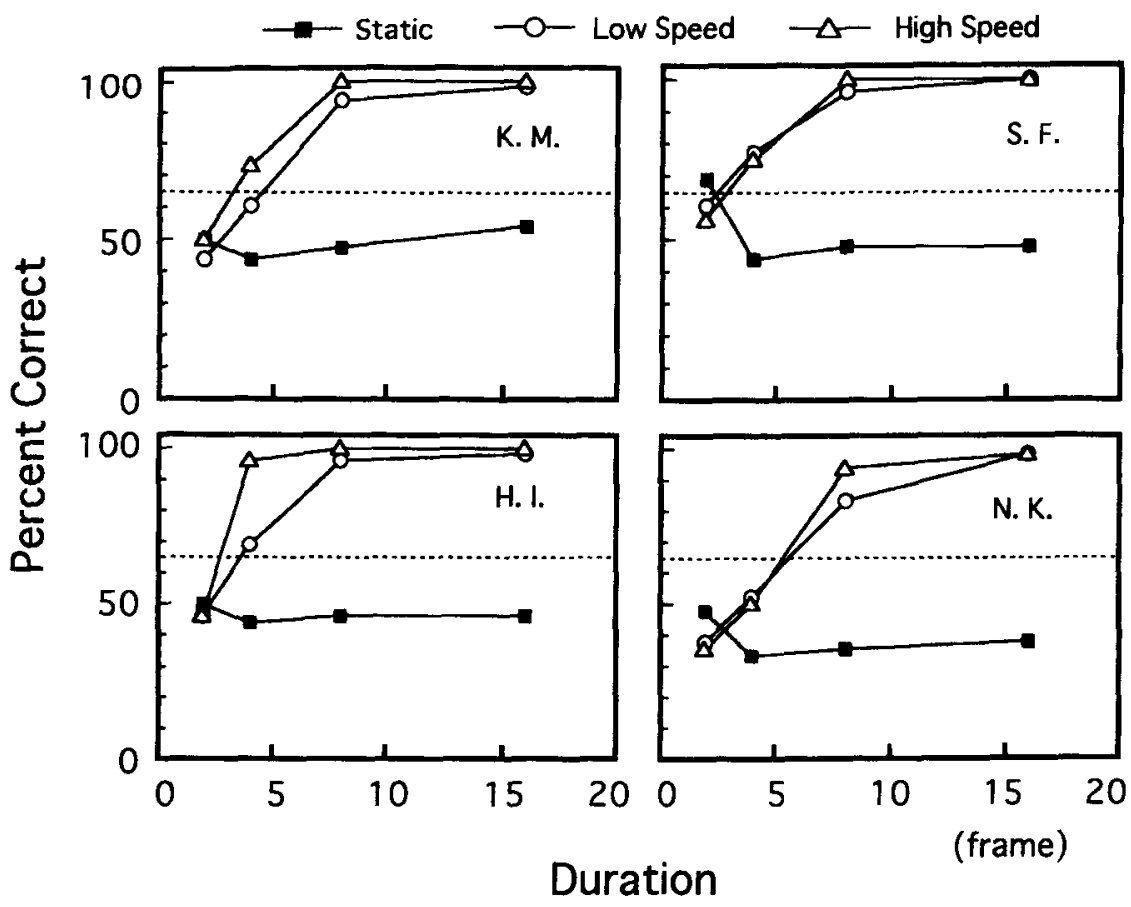

Figure 8. Percent correct under each frame condition. Circles, squares, and triangles represent the data under high-speed, low-speed, and static conditions, respectively. The dotted lines in the figure indicate the upper limit of random probability $(p<.05)$.

and a finer resolution), or tasks should be more difficult so that the performance cannot easily reach ceiling.

\section{GENERAL DISCUSSION}

In these three experiments, it was investigated whether or not the human visual system could detect the 3-D structure of opening from expanding motion - that is, whether or not an observer could perceive the surface layout of the surround from optic flow during forward motion. Experiments 1 and 2 demonstrate the validity of perception of 3-D structure from expanding motion. The required duration was investigated in Experiment 3, and the results show that sensitive observers could roughly detect the 3-D structure with four-frame $(73-\mathrm{msec})$ presentation and that eight frames were enough also for the other observer. It can be concluded that expanding motion is useful for the visual system in the detection of an opening's 3-D structure while one is in forward motion.

One possible hypothesis for the detection mechanism is that the differences of local expansion rates are a cue to depth and that their global distribution would determine the perceived 3-D structure. Smooth changes of the rate gradient might lead to surface and slant perception. This kind of approach, in which detecting local relative motion contributes to detecting an object's 3-D structure, has been described by Koenderink (1986). He suggests that there are four basic motion components and that local relative expansion components can be used for detecting the 3-D structure of objects. A model for the mechanism that detects relative expansion components has been proposed by Regan and Hamstra (1993). In addition, Ito and Matsunaga (1990) have suggested that a plane with higher expansion rate is perceived to be nearer. These researchers seem to suggest that the local expansion component is detected over the entire retina: when the expanding rate is lower at the center than at the peripheral field, a concave (opening) may appear, while on the other hand, when the expanding rate is higher at the center, a convex (obstacle) may appear. Furthermore, if the flow indicates an opening, and if the right and left parts of the visual field expand at a higher rate than do the upper and lower parts, the path will be narrow in the horizontal orientation (the side walls are near).

Another possible explanation is that dots having the same global expansion rate might form the shape of the vertical section. Andersen's (1989) report suggested the possibility of elements' grouping across the visual field, based on global expansion rate. If the grouping across the field occurred in the display used here, it would have indicated the shape of the vertical section.

The former explanation would be inconsistent with Andersen's (1989) report. As noted earlier, in his display, although local expansion components were broken by overlapping dot planes, subjects could detect three dot planes that were at separate depths. This suggests that detecting local expansion is unnecessary for the process of recovering 3-D structure from motion. On the other hand, the latter explanation would seem to have little efficiency in reality, because actual openings and obstacles 
have quite complicated 3-D structures in comparison with those of tunnels and it would seem inconvenient to cut the opening in slices, each of which would have the same expansion rate. Representations composed of depth spreading (distances to objects' surfaces) would be more convenient for humans to decide the course, avoiding a possible collision. Interactions between local and global motion processes play an important role in the recovery of 3-D structure from expanding motion.

\section{REFERENCES}

ANDersen, G. J. (1989). Perception of three-dimensional structure from optic flow without locally smooth velocity. Journal of Experimental Psychology: Human Perception \& Performance, 15, 363371.

Braunstein, M. L., \& Tittle, J. S. (1988). The observer-relative velocity field as the basis for effective motion parallax. Journal of Experimental Psychology: Human Perception \& Performance, 14, 582590.

GiBson, J. J. (1950). The perception of the visual world. Boston: Houghton Mifflin.

GiBSON, J. J. (1979). The ecological approach to visual perception. Boston: Houghton Mifflin.

Ito, H., \& Matsunaga, K. (1990). Relative distance perception through expanding and contracting motion and the role of propriospecific information in walking. Ecological Psychology, 2, 113-130.

KOENDERINK, J. J. (1986). Optic flow. Vision Research, 26, 161-180.
ONo, H., \& Steinbach, M. J. (1990). Monocular stereopsis with and without head movement. Perception \& Psychophysics, 48, 179-187. REGAN, D., \& BEVERLEY, K. I. (1978a). Illusory motion in depth: Aftereffect of adaptation to changing size. Vision Research, 18, 209212.

Regan, D., \& Beverley, K. I. (1978b). Looming detectors in the human visual pathway. Vision Research, 18, 415-421.

Regan, D., \& Hamstra, S. J. (1993). Dissociation of discrimination thresholds for time to contact and for rate of angular expansion. $\mathrm{Vi}$ sion Research, 33, 447-462.

Rogers, B., \& Graham, M. (1979). Motion parallax as an independent cue for depth perception. Perception, 8, 125-134.

SChiff, W., CAviness, J. A., \& Gibson, J. J. (1962, June 15). Persistent fear responses in rhesus monkeys to the optical stimulus of "looming." Science, 136, 982-983.

Simpson, W. A. (1988). Depth discrimination from optic flow. Perception, 17, 497-512.

ToDD, J. T. (1981). Visual information about moving objects. Journal of Experimental Psychology: Human Perception \& Performance, 7 , 795-810.

ULLMAN, S. (1979). The interpretation of visual motion. Cambridge, MA: MIT Press.

ULLMAN, S. (1984). Maximizing rigidity: The incremental recovery of 3-D structure from motion. Perception, 13, 255-274.

Wallach, H., \& O'Connell, D. N. (1953). The kinetic depth effect. Journal of Experimental Psychology, 45, 205-218.

(Manuscript received January 29, 1993; revision accepted for publication May 23, 1995.) 